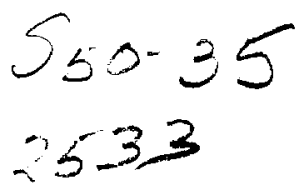

N94-32470

\title{
THE TRUSTWORTHY DIGITAL CAMERA: RESTORING CREDIBILITY TO THE PHOTOGRAPHIC IMAGE
}

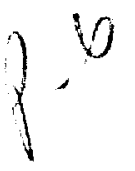

\author{
Gary L. Friedman \\ Technical Group Leader \\ Advanced Information Systems \\ Jet Propulsion Laboratory \\ California Institute of Technology \\ Pasadena, CA 91109
}

\section{Introduction}

The increasing sophistication of computers has made digital manipulation of photographic images (as well as other digitally-recorded artifacts, such as audio and video) incredibly easy to perform and, as time goes on, increasingly difficult to detect. Today, every picture appearing in newspapers and magazines has been digitally altered to some degree, with the severity varying from the trivial (cleaning up "noise" and removing distracting backgrounds) to the point of deception (articles of clothing removed, heads attached to other people's bodies, the complete rearrangement of city skylines). As the power, flexibility and ubiquity of image-altering computers continues to increase, the well-known adage that "the photograph doesn't lie" will continue to become an anachronism.

A solution to this problem comes from a concept called Digital Signatures, which incorporates modern cryptographic techniques to authenticate electronic mail messages. [1] [2] ("Authenticate" in this case means you can be sure that the message has not been altered, and that the sender's identity has not been forged.) The technique can serve not only to authenticate images, but also to help the photographer retain and enforce copyright protection when the concept of "electronic original" is no longer meaningful.

\section{Background on Digital Signatures}

The concept of a digital signature builds upon a recent encryption technique called "Public Key Encryption" [3]. Older encryption/decryption schemes require that both the sender and receiver possess the same secret "key": the sender uses the key to transform the text message into ciphertext, and the receiver uses the same key to perform an inverse transformation on the ciphertext, revealing the original text message. If the correct key transforms the ciphertext into unreadable garbage, it is reasonable to conclude that either the wrong key is being used, the message has been altered, or the sender has been impersonated by someone ignorant of the correct key. The historic drawback to this secret key encryption scheme has been in the secure distribution of keys; key disclosure must occur out-ofband, either transmitted via an expensive alternate path or arranged when sender and receiver were proximate.

Public key encryption techniques differ in that they enable the recipient of a message to decrypt it using a key that is different from the one used by the sender to encrypt it. All public key cryptography is based on the principle that it is easy to multiply two large prime numbers together, but extremely difficult (taking perhaps centuries using today's supercomputers) to work backwards and uncover the factors that could have been used to generate the resulting number.

Public Key Encryption employs two different keys: a private key, which is held by the more security conscious party, and a corresponding public key, which need not be kept secret. The public key is generated based upon the private key, making the pair unique to each other.

The public key scheme is illustrated in Figure 1 and works as follows: to send a secret message that only the recipient can read, the recipient would first make his/her public key known to the sender through any non-secure medium, such as a letter, a telephone conversation, or a newspaper ad. Anyone wishing to send a secure message would encrypt the message using this public key and send it to the recipient. The recipient, having sole possession of the corresponding private key, is the only one able to decrypt the message. The need to transmit a secret key that both parties must possess beforehand has been eliminated. The tradeoff in this case is that, although only the recipient can read the message, anyone who obtains the public key can send a message with anonymity. 1

\footnotetext{
1 The described scenario can also be used as the first step in a process of exchanging secret keys to allow for conventional secure message transmission, eliminating any of the drawbacks of the one-way authenticatability. [1], [4]
} 


\section{SUMMARY AND CONCLUSIONS}

This paper has briefly reviewed features important to high-capacity photographic image data capture, classification, compression, storage, retrieval, and display. Also described was a NASA-KSC space shuttle ground processing prototype IDBMS under development which provides knowledgebased assistance for image classification and retrieval. Finally, a design for a networked PC-LAN IDBMS was presented. A conclusion reached from reviews of the prototype system is that it has distinct advantages over the present manual system and cost efficiencies will result as the system is implemented. Further, commercial potential exists for this integrated technology.

\section{ACKNOWLEDGEMENT}

This research project is sponsored under a NASA-KSC/UCF Cooperative Agreement (NAG-100058). Appreciation is expressed to NASA-KSC's Nancy Sliwa, Astrid Heard, and William Helms of the Advanced Projects Office for their vision and support.

\section{REFERENCES}

[1] Ragusa, J.M. and Orwig, G, "Expert systems and imaging: NASA's start-up work in intelligent image management," Expert Systems, vol. 2, no. 3, 25-30, Winter 1990.

[2] Ragusa, J.M. and Orwig, G., "Attacking the information access problem with expert systems," Expert Systems, vol. 2, no. 4, 26-32, Spring 1991.

[3] Ragusa, J.M. and Wielgos, R., "Using expert systems to interface relational and object-oriented databases for a NASA space shuttle applications," Heuristics: The Journal of Knowledge Engineering, vol. 4, no. 3, 1-10, Fall 1991.

[4] Coopers \& Lybrand, Information \& Image Management: The Industry \& the Technology. New York: Author, 1987.

[5] Thompson, D., "Imaging meets expert systems," AI Expert, vol. 6, no. 11, 24-32, Nov., 1991.

[6] Harmon, P., Maus, R., and Morrissey, W., Expert Systems: Tools \& Applications. New York: John Wiley, 1988.

[7] Liebowitz, J., An Introduction to Expert Systems. Santa Cruz, CA: Mitchell Publishing, 1988.

[8] Mockler, R.J. and Dologite, D.G., Knowledge-Based Systems: An Introduction to Expert Systems. New York: Macmillan, 1992.

[9] McGraw, K.L. and Harbison-Briggs, K., Knowledge Acquisition: Principles and Guidelines. Englewood Cliffs: Prentice Hall, 1989.

[10] Scott, A.C., Clayton, J.E., and Gibson, E.L., A Practical Guide to Knowledge Acquisition. Reading: Addison-Wesley, 1991.

[11] Besser, H., "Visual access to visual images: The U Berkeley image database project," Library Trends, vol. 38, no. 4, 787-798, Winter 1990. 
The process described above can also be implemented "backwards" to great advantage. In a second scenario, it is the sender who maintains possession of the private original plaintext message, and then encrypting the hash using the sender's private key, as shown in Figure 2. The result is a second digital file (referred to as a

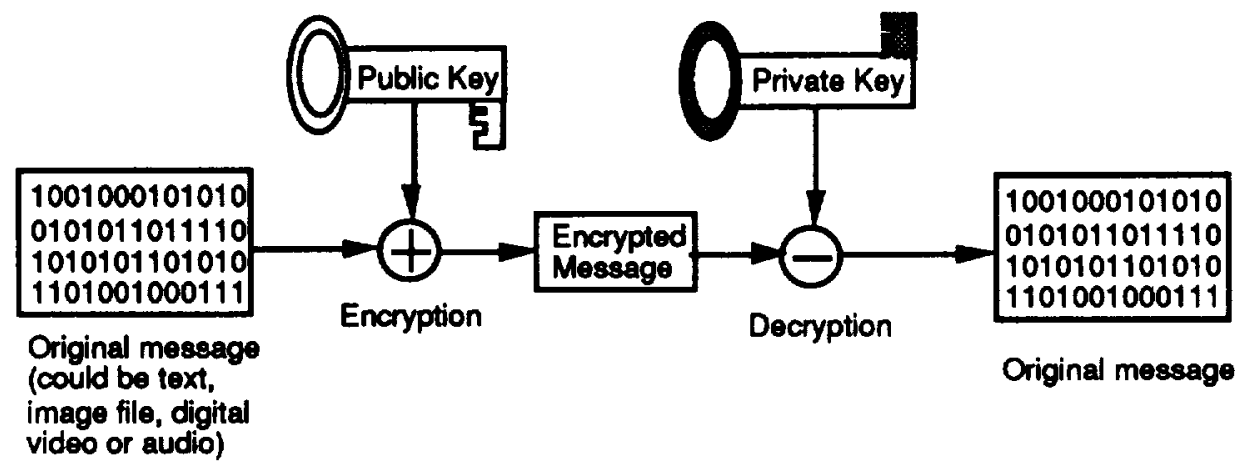

Floure 1: With Public Key Encryption, the encoding (public) key and decoding (private) keys are different, and it is computationally difficult to derive one given the other. To send a message that only the receiver can read, the non-secret public key is used to encrypt; the secret private key is used to decrypt. Encrypting with the private key forgoes confidentiality in favor of authenticability: if the public key can decode it, then only the one holding the private key could have generated the message. signature) which accompanies the original plaintext message. To emphasize: THE ORIGINAL MESSAGE IS UNTOUCHED; only the message's hash is encrypted. This way the original file can be read by all, yet if you wish to authenticate it you can decrypt the message's unique digital signature using the public key. If the decrypted digital signature and an independent hash on the file in question match, both the integrity of the message and the authenticity of the sender can be assured.

key, and anyone who has the widely disseminated corresponding public key could decrypt this message. Although this procedure no longer performs the traditional function of encryption (which is to provide confidential communication between two parties), it does provide a way to insure that messages have not been forged: only the private key could have produced a message that is decipherable by the corresponding public key.

This gives us the foundation for message authentication: if the private key remains private, then only the private key holder can produce messages decipherable by the public key. Furthermore, it is extremely difficult to reverse-engineer the public key and ascertain the original private key. Without knowledge of the private key, a counterfeit message cannot be forged.

Digital signatures build upon these public key cryptographic techniques and allow you to authenticate the contents of the message as well as the identity of the sender, without obscuring the original message. The signatures are produced by creating a hash ${ }^{2}$ of the

\footnotetext{
${ }^{2} \mathrm{~A}$ hash is a mathematical function which maps values from a large domain into a smaller range. For example, a checksum is a simple kind of hash. A more complex example of a hash algorithm would involve dividing a binary file into a collection of, say, 16 Kilobit pieces and performing a cumulative Exclusive OR function between successive pieces produces a simple 16 Kilobit "hash" which is smaller than the original file yet is practically unique to it. (Many more complex and secure transformations are also possible.) Changing a single bit in the original message produces a very different hash output; and
}

This digital signature technique is very general; it can be applied not only to 1-dimensional symbolic text (such as electronic mail) but also to any n-dimensional digital pattern (such as digital video, digital audio, and/or digital holograms).

Standard digital cameras are filmless; they sense light and color via an electronic device (such as a Charge Coupled Device (CCD)), and produce as output a computer file that describes the image using 1 's and 0 's arranged in a meaningful, pre-defined format. Often this digital image file is stored on a small mass-storage medium inside the camera itself (such as floppy disk or magneto-optical disk) for later transference to a large computer. Alternatively, the image file can be sent directly to the computer via a transmission medium. Once inside the computer it then can be read and then easily altered in any number of different ways.

In the proposed digital camera (Figure 3) we wish to authenticate the initial image file as it emerges from the camera. To accomplish this, the camera produces two output files for each captured image as shown in Figure

reverse engineering a message so it will have a given hash value and also make sense to the reader is virtually impossible. A digital signature can then be created by encrypting the hashing output using the sender's private key.

\section{Digital Cameras}


4: the first is an all-digital industry-standard file format representing the captured image. The second would be an encrypted "digital signature" produced by applying originally produced. If on the other hand at least a single bit is different, the two hashes will not even closely match and the image's integrity will not be affirmed.

If the technique is to be effective (i.e., no false positives or false negatives) and extended to larger data sets such as digital audio, digital video or even digital holograms, we must build upon the accomplishments of the computer mass storage industry, which has already achieved the ability to store and deliver extremely large binary data sets without errors. Analog

the camera's unique private key (embedded within the camera's secure microprocessor) to a hash of the captured image file, using the procedure described in [4]. It is the responsibility of the user to keep track of both files once they leave the camera, since both are required to authenticate the image.

Once the digital image file and the digital signature are generated by the camera and stored in computer memory, the image file's integrity can later be affirmed by using a public key decoding program, which can be freely distributed to users and certification authorities via conventional software distribution techniques. This verification program (illustrated in Figure 5), which has no knowledge of either the public or private keys, takes as input the digital image file in question, its accompanying digital signature file, and the public key which is unique to the originating camera. (It is perfectly reasonable to have the public key double as the camera's serial number.) The program then calculates its own hash on the digital image file (the hashing algorithm need not be kept a secret), and uses the public key to decode the digital signature to reveal the hash originally calculated by the camera at the time the image was taken (Figure 6). If these two hashes match, it is certain to any required degree that the digital image in question is indeed identical to what the camera techniques (such as audio cassette tape or the NTSC encoding on today's video tape formats) or noncorrected digital formats (such as the popular audio compact disc (CD), which is so unreliable that $C D$ player manufacturers now employ "over-sampling" to combat the problem of missed bits) introduce a large amount of errors upon playback that are normally imperceptible to the viewer or listener, but are intolerable for the purposes of image authentication.

\section{Measures of Protection}

The scheme as described above is resistant to forgery attempts since the secret private key (which is known
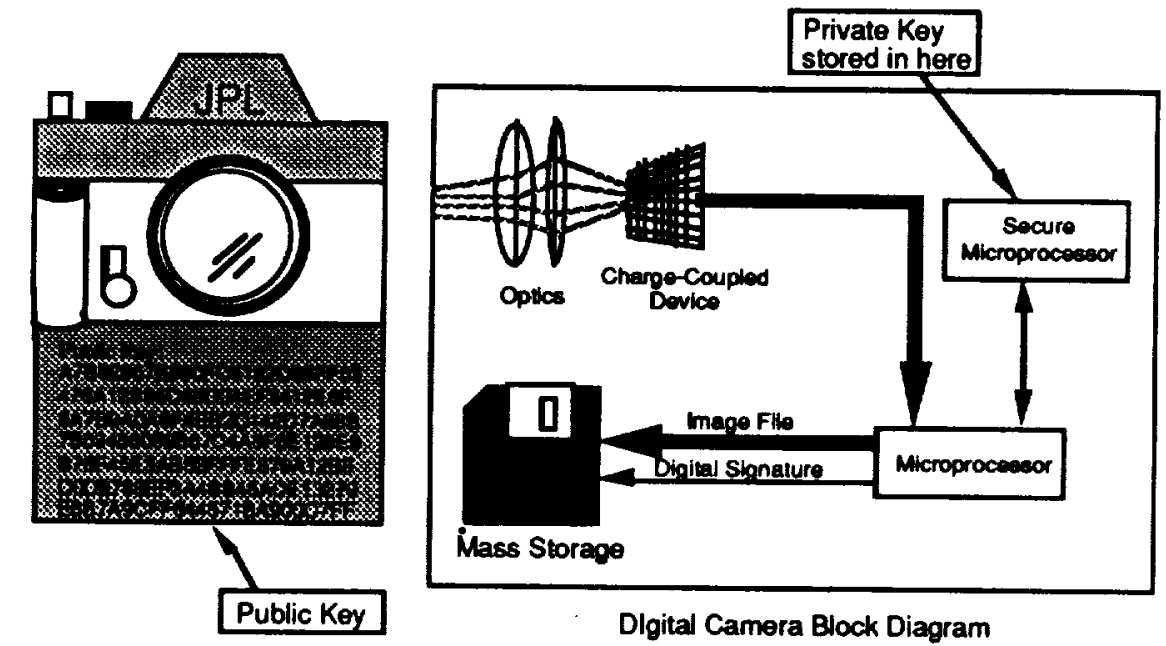

Dlgital Camera Block Diagram

Figure 3: The Trustworthy Digital Camera starts with a digital sensor instead of film. and delivers the image directly in a computer-compatible format. The secure microprocessor responsible for the encryption of the digital signature is programmed with the private key at the factory. The public key necessary lor later authentication appears in the image's border as well as on the camera body. 
only by the camera's manufacturer) is embedded in a probe-proof microprocessor which itself is deeply integrated into the camera's system (Figure 1). Even if some adept pirate were to dissect the camera and replace

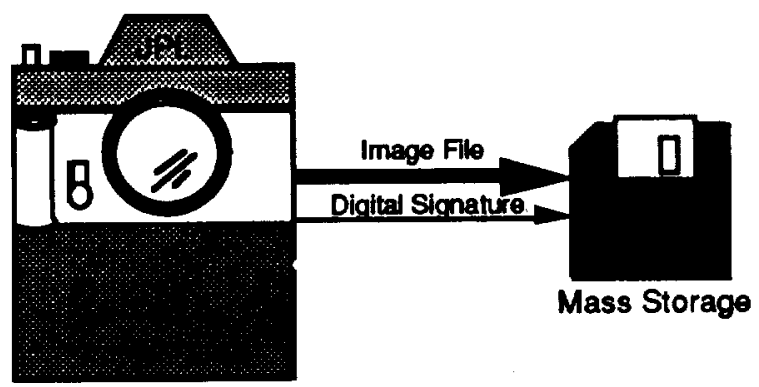

Figure 4: When a single photo is taken, two files are produced: a standard digital image file, and an encrypted digital signature. The files can be stored on a variety of media, such as a Write-Once-Read-Many (WOAM) CD or the computer's mass storage device. The image can then be accessed and used just as any other computer data.

the chip with one containing a homebrew key, the digital signature produced thereafter would not be decodable by any public key published by the manufacturer.

The advantages to freely distributing the verification software and valid public keys are great; with the software freely available verification can become commonplace and routine. No special certification authority need be called in for routine checks, no fees are required, no big fuss is made, and no bad-faith climate amongst the parties involved need be created as a result of being challenged. But the mass distribution of verification software does carry one danger: it would be easy for someone to create a bogus program that looks, behaves, and has the same file length as the genuine verification software, with the only difference being it always proclaims a "match" regardless of the integrity of the image being verified. With the software freely and widely available this is not a large risk, as additional copies can be easily obtained from multiple sources and a best 2-out-of-3 scheme can be employed. When the stakes are high and it is extremely important that the verification software be known to be genuine, an independent certification authority or the manufacturer could then be called in to provide their own copies of the software and their own lists of public keys at the time of verification.

The algorithms and private key necessary for encrypting the additional digital signature file from within the camera are to be embedded inside a new breed of secure microprocessors whose ROM contents cannot be observed outside of the factory. Because the private key used for encryption is hard-coded into this chip by the manufacturer (who must then ensure the private key remains secret), credibility of the camera's output becomes an extension of that of the manufacturer; a digital signature from the camera can be considered to be just as reliable and secure as if the signature had been generated by the manufacturer. 3

Each camera should possess its own unique pair of private and public keys, with the private key etched into the camera's secure microcontroller and the public key stored in three places: in a public key list kept by the manufacturer, on the camera body itself (which can then also double as the camera's serial number), and in the colorful border that contains more data about the captured image (see "A Special Border" section below for more details on this idea). Assigning unique keys to each camera has the benefit of avoiding instant obsolescence which would occur if only one private key were used for all cameras, and that key were

Flgure 5: To authenticate the Image, public domain verification software is run on a standard computer plattorm. The program takes as input the image file in question, the digital signature, and the camera's serial number (which doubles as its public key).

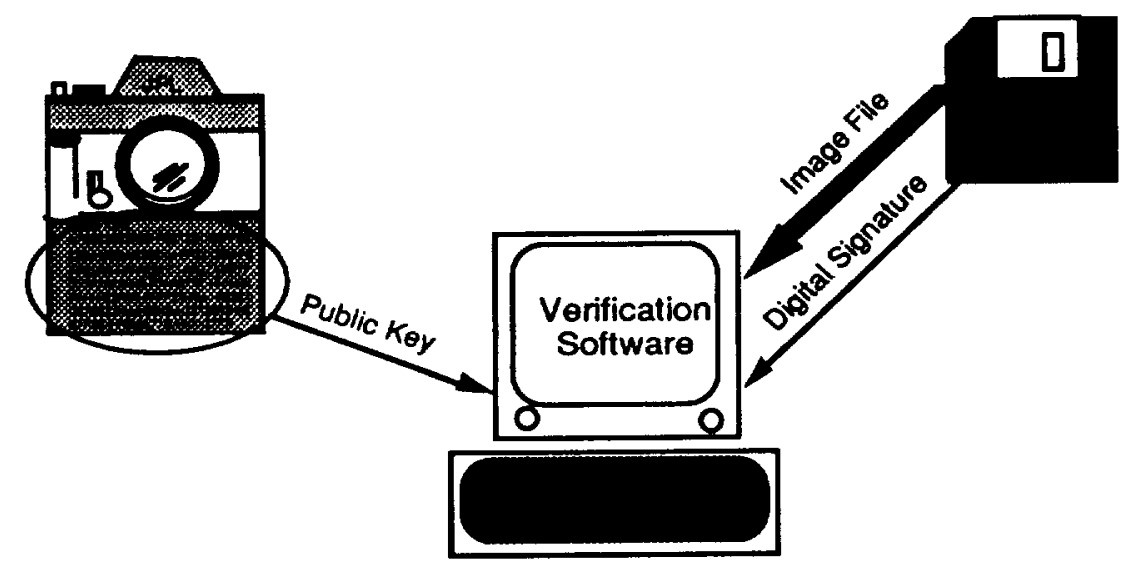

to be compromised. An even higher level of security

3 Any company involved with the development of a Trustworthy Digital Camera would have to address the issue of liability, for if the security of the private key were ever to be compromised (for example by a disgruntled employee who steals a private key and uses it to generate false authenticatable inages), the lawsuits brought on as the result of a false positive would necessitate significant insurance coverage. 


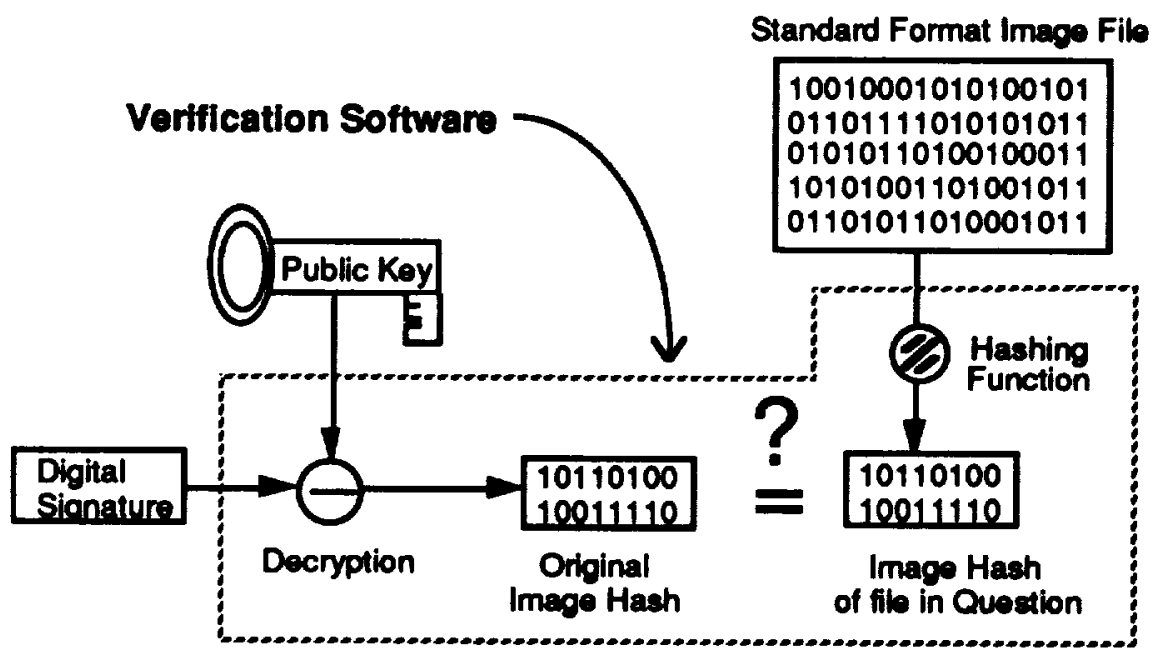

Flgur 6: The verification sotwware computes its own hash of the image in question, and compares it to the original hash which has been decrypted using the public key. If the image in question has not been manipulated, the docrypted digital signature and the program's own hashing function will match, resulting in an authentication. If oven a single bit is different, the two hashes will not even closely match, yiolding an authentication fallure.

would occur if the manufacturer were to destroy all records of the private key once the cumern is produced. (At that point the private key is no longer needed.) This would eliminate the possibility of compromise via industrial espionage or theft.

Finally, regular and free distribution of all valid public keys is desirable to defeat a counterfeiter who has learned of the encryption algorithm employed and has written a program to produce digital signatures based on his own private key. Decoding these forgeries would require the use of a public key not generated by the manufacturer. Freely distributing updated public key lists would make it easy to identify and thwart such attempts.

Uses

The single most obvious use of a trustworthy camera would be in situations where proof of image authenticity is necessary; such as for legal evidence or insurance claims. The inevitable transition to digital cameras and electronically-transmitted images will also make it more difficult for the professional photographer to protect his or her image copyright, since with electronic cameras there is no "original" to control, and works stored in computer format tend to proliferate faster and with less control from the author than the traditional distribution method (which places image control in the hands of whoever holds the original negative or transparency). Just as it is common practice today to obtain model releases for any published picture containing a recognizable face, it is foreseeable that no electronic image in the future will be published without first having authenticated the image using the digital signature of the camera which was or is registered to the photographer.

This technique need not be limited to still digital images. Because digital signatures can be used to verify any block of digital data, it can also be engineered into digital video cameras and digital audio tape recorders. In both these devices, a digital signature can be generated and recorded onto the medium each time the recording process stops or pauses; this way each sound byte or video "take" is hashed, encoded and written at the time it's created.

\section{A Special Border}

Since the proposed camera is being initially targeted towards legal authentication, a few additional features can be implemented to better serve this use. A brightlycolored border could automatically be generated as part of each captured image file. Within the border would appear textual information about the image: the date and time it was taken, the ambient light level seen by the camera at the time of exposure, the original color temperature of the scene, the software version of the camera's firmware, the camera's serial number, the focusing distance of the lens at the time of exposure, a unique sequence number, and (when the technology allows for a Global Positioning System (GPS) receiver to be built into the camera) the geographical coordinates of the camera, indicating where in the world you were when the picture was taken. The ambient light level and 
color temperature readings would be useful for getting a feel for exactly what the scene was like at the time of exposure; something a sensitive optical element might inadvertently hide via automatic exposure and color correction. The lens' focused distance is there to help detect potential abuse of the trustworthy camera: taking close-up pictures of a modified photo and trying to pass it off as an unaltered original. Since all these textual data in the colored border are part of the authenticated image file, their credibility is also upheld when authenticated by the verification software.

The accuracy of the date and time information would again be the responsibility of the secure microprocessor, in addition to being able to keep its programming a secret, it also would have a lithium battery powering a system clock that was set to Universal (Greenwich Mean) Time at the time of manufacture. If the timer circuit ever fails or is tampered with, the system will be programmed to fill the time and date fields with XXXX's, eliminating the chance of a random time stamp being mistaken for the actual time.

\section{Higher Level of Security}

Although the proposed Trustworthy Digital Camera offers a satisfactory level of security, nevertheless there still exists a small possibility that a determined saboteur will be able to crack the camera's private key given an extended amount of time. (No cryptographic scheme will protect your data forever; given sufficient time, advancements in code breaking or improved computer horsepower will be enough to render any given level of cryptographic protection obsolete.) If the discovered private key were then to be published, it would allow an individual to generate authentic-looking digital signatures on altered image files, essentially undermining the credibility offered by the compromised camera. (The security level of other cameras in use, and of images taken with those cameras, will still remain high.)

Because of this risk, it would be wise for a manufacturer of such cameras to regularly upgrade and enhance the sophistication of the encryption implementation as newer camera models are introduced, typically using longer encryption/decryption key lengths and improved encryption/ decryption algorithms. It is expected that evolving verification software (the public domain software component of this authentication scheme which is freely distributed) will then be designed to recognize, identify and authenticate all previous versions.

Because the encryption details must necessarily be changed often (depending on the technological capabilities of the day), no single image format, key length or digital signature algorithm is being specified in this disclosure 4 .

\section{Conclusion}

The Trustworthy Digital Camera is an application of existing technology toward the solution of an evermore-troubling social problem, the eroding credibility of the photographic image. Although it will always be possible to lie with a photograph (using such timehonored techniques as false perspective and misleading captions), this proposed device will prevent the explosion of very capable personal computers from driving up the incidence of doctored photographs being passed off as truth.

The research described in this paper was carried out by the Jet Propulsion Laboratory, California Institute of Technology, under a contract with the National Aeronautics and Space Administration.

\section{Bibliography}

[1] "A Public Key Cryptosystem and a Signature Scheme Based on Discrete Logarithm", IEEE

Transactions on Information Theory, Vol. IT-31 no. 4, (July 1985), Taher El Gamal, pp. 473-81.

[2] "A Method for Obtaining Digital Signatures and Public-Key Cryptosystems", Communications of the ACM, Vol. 21 no. 2 (February, 1978), R.L. Rivest, A. Shamir, L. Adleman, pp. 120-26.

[3] "New Directions in Cryptography", IEEE

Transactions on Information Theory, Vol. IT-22 no. 6 (November, 1976), Whitfield Diffie and Martin E. Hellman, pp. 644-54.

[4] "The Proposal for a U.S. Standard for Digital Signature Encoding", IEEE Spectrum, August, 1992, Dennis K. Branstad, pp. 30.

[5] "8051 Tackles Secure Smart-card Applications", EDN, December 10, 1992, pp. 46-48.

[6] "Photography by the Numbers", BYTE, January 1993, Howard Eglowstein, pp. 241-244.

${ }^{4}$ Although the National Institute of Standards and Technology's (NIST's) proposed Digital Signature Standard (DSS) was in mind when this method was conceived, adherence to it, its algorithms, formats, or royalties is not required for implementation. 
